\title{
Super-Massive Neutron Stars
}

\author{
Paulo C. C. Freire \\ N.A.I.C., Arecibo Observatory, HC3 Box 53995, PR 00612, U.S.A.; pfreire@naic. edu
}

\begin{abstract}
We present here the results of Arecibo timing of PSR B1516+02B, a 7.95-ms pulsar in a binary system with a $\sim 0.17 M_{\odot}$ companion and an orbital period of 6.85 days located in the globular cluster M5. The eccentricity of the orbit $(e=0.14)$ has allowed a measurement of the rate of advance of periastron: $\dot{\omega}=(0.0136 \pm 0.0007)^{\circ} \mathrm{yr}^{-1}$. It is very likely that the periastron advance is due to the effects of general relativity; the total mass of the binary system is $(2.14 \pm 0.16) M_{\odot}$. The small measured mass function implies, in a statistical sense, that a very large fraction of this total mass is contained in the pulsar: $M_{p}=\left(1.94_{-0.19}^{+0.17}\right) M_{\odot}(1-\sigma)$; there is a $5 \%$ probability that the mass of this object is below $1.59 M_{\odot}$. With the possible exception of PSR J1748-2021B, this is the largest neutron star mass measured to date. When combined with similar measurements made previously for Terzan $5 \mathrm{I}$ and J, we can exclude, in a statistical sense, the "soft" equations of state for dense neutron matter, implying that matter at the center of a neutron star is highly incompressible. There is also some evidence for a bimodal distribution of MSP masses, the reasons for that are not clear.
\end{abstract}

Keywords: Millisecond Pulsars; Binary Pulsars; Precision Timing, Neutron Stars, Equation of State PACS: 97.60.Gb;97.60.Jd;97.60.-s;97.80.-d;

\section{TIMING OF M5B}

Over the past 20 years, more than 130 pulsars have been discovered in globular clusters (GCs $\sqrt{1}$. Among the first discoveries were PSR B1516+02A and PSR B1516+02B [1]. Both of these millisecond pulsars (MSPs) are located in the GC NGC 5904, also known as M5; for this reason we will refer to them as M5A and M5B. The latter is a $7.95-\mathrm{ms}$ pulsar in a binary system with a $\sim 0.17-M_{\odot}$ companion and an orbital period of 6.86 days. At the time of its discovery, this was the MSP with the most eccentric orbit known $(e=0.14)$. In the Galaxy, $80 \%$ of MSPs are found to be in binary systems, and with a single exception (PSR J1903+0327, see David Champion's contribution to these Proceedings) they are in low-eccentricity orbits with white dwarf (WD) companions. In GCs, gravitational interactions with neighboring stars can greatly increase the eccentricity of these binary systems [2]; the orbital eccentricity of M5B is $\sim 10^{4}-10^{5}$ times larger than that of Galactic MSP-WD systems with similar orbital periods. When Anderson et al. (1997) published the timing solutions of M5A and B, they used the eccentricity of M5B to detect its periastron advance, but the large relative uncertainty of the measurement did not allow any astrophysically useful constraints on the total mass of the binary.

In [4], Freire et al. report the results of recent (2001 to 2007) L-band observations of these two pulsars. The first 2001 observations were part of an Arecibo search

\footnotetext{
${ }^{1}$ See Scott Ransom's review, in these Proceedings. For an updated list, see http://www2.naic.edu/ pfreire/GCpsr.html
}

for pulsars in GCs, which found a total of 13 new MSPs [5]. Three of these were found in M5, subsequent observations of this $\mathrm{GC}$ were made chiefly with the aim of timing the new discoveries. However, both M5A and M5B are in the same radio beam as the new pulsars. They are clearly detectable in the L-band data, allowing for timing of much better (M5A) or comparable (M5B) quality to that obtained at $430 \mathrm{MHz}$. Including those previous data, this provides a much longer total timing baseline (18 years) and much improved timing parameters.

\section{Observations, data reduction and timing}

The L-band observations started in 2001 June. Until 2003, we used the "old L-Wide" receiver in the Gregorian Dome $\left(T_{\text {sys }}=40 \mathrm{~K}\right.$ at $\left.1400 \mathrm{MHz}\right)$. Since 2003 February, we have been using the current "L-Wide" $\left(T_{s y s}=25 \mathrm{~K}\right.$ at $1400 \mathrm{MHz}$. The Wide-band Arecibo Pulsar Processors (WAPPs, [6]) made a 3-level digitization of the voltages of a $100 \mathrm{MHz}$-wide band for both (linear) polarizations, correlating them for a total of 256 lags. These were then integrated for a total of $64 \mu \mathrm{s}$ and the results of both polarizations added in quadrature and written to disk. At first, only one WAPP was available. In this case we centered the observing band at $1425 \mathrm{MHz}$. After 2003, three more WAPPs became available, and we started observing simultaneously at 1170, 1420 and $1520 \mathrm{MHz}$, thanks to the wide frequency coverage of the new L-wide receiver.

The lags were then Fourier transformed to generate power spectra. These were then dedispersed at the known DM of these pulsars and folded modulo their spin pe- 
riods using the PRESTO pulsar software package 2. We then cross-correlated the resulting pulse profiles with the average pulse profile in the Fourier domain [7] to obtain topocentric times of arrival (TOAs). These were then analyzed with TEMPC 3 , together with the TOAs derived from the old 430-MHz observations made from 1989 to 1994. We used the DE 405 Solar System ephemeris [8] to model the motion of the Arecibo 305-m Radio Telescope relative to the Solar System Barycenter.

The resulting timing parameters are presented in [4]. Astrophysically, the most important new parameter is the rate of advance of periastron for M5B: $\dot{\omega}=(0.0136 \pm$ $0.0007)^{\circ} \mathrm{yr}^{-1}$.

\section{Binary, pulsar and companion masses}

As argued in [4], the $\dot{\omega}$ is solely due to the effects of general relativity. In this case, we can estimate the total mass of a binary system:

$$
M=\left(\frac{P_{b}}{2 \pi}\right)^{5 / 2}\left[\frac{\left(1-e^{2}\right) \dot{\omega}}{3}\right]^{3 / 2}\left(\frac{1}{T_{\odot}}\right)
$$

where $T_{\odot} \equiv G M_{\odot} / c^{3}=4.925490947 \mu$ s. Using the $\dot{\omega}$ above, we obtain $M=(2.14 \pm 0.16) M_{\odot}$. For the nominal $\dot{\omega}$ and a median inclination of $60^{\circ}$, the mass of the companion is $0.166 M_{\odot}$ and the mass of the pulsar is $1.98 M_{\odot}$. This is well above all the neutron star masses that have been precisely measured to date.

We calculated a 2-D probability distribution function (pdf) for the mass of the pulsar and the mass of the companion, assuming that the pdf for $\dot{\omega}$ is a Gaussian with the half-width equal to the $1-\sigma$ uncertainty of the observed $\dot{\omega}$ and an a priori constant probability for $\cos i$. The two-dimensional pdf is then projected in both dimensions, resulting in 1-D pdfs for the mass of the pulsar and the mass of the companion. These are displayed graphically in Fig. 1. The pulsar definitely has a mass smaller than $2.35 M_{\odot}$, and the companion has a mass larger than $0.13 M_{\odot}$, the median and $1-\sigma$ limits for the pulsar and companion mass are $1.94_{-0.19}^{+0.17} M_{\odot}$ and $0.164_{-0.022}^{+0.10} M_{\odot}$ respectively. There is a $99 \%, 95 \%$ and $90 \%$ probability that the pulsar is more massive than $1.19,1.59$ and $1.69 M_{\odot}$. There is a $1.3 \%$ probability that $i$ is low enough to make the neutron star mass fall within the range of NS masses observed in double neutron star (DNS) systems: from $1.20 M_{\odot}$ measured for the companion of PSR J1756-2251 [9] to $1.44 M_{\odot}$ measured for PSR B1913+16 [10].

\footnotetext{
2 http://www.cv.nrao.edu/ sransom/presto

3 http://www.atnf.csiro.au/research/pulsar/tempo/
}

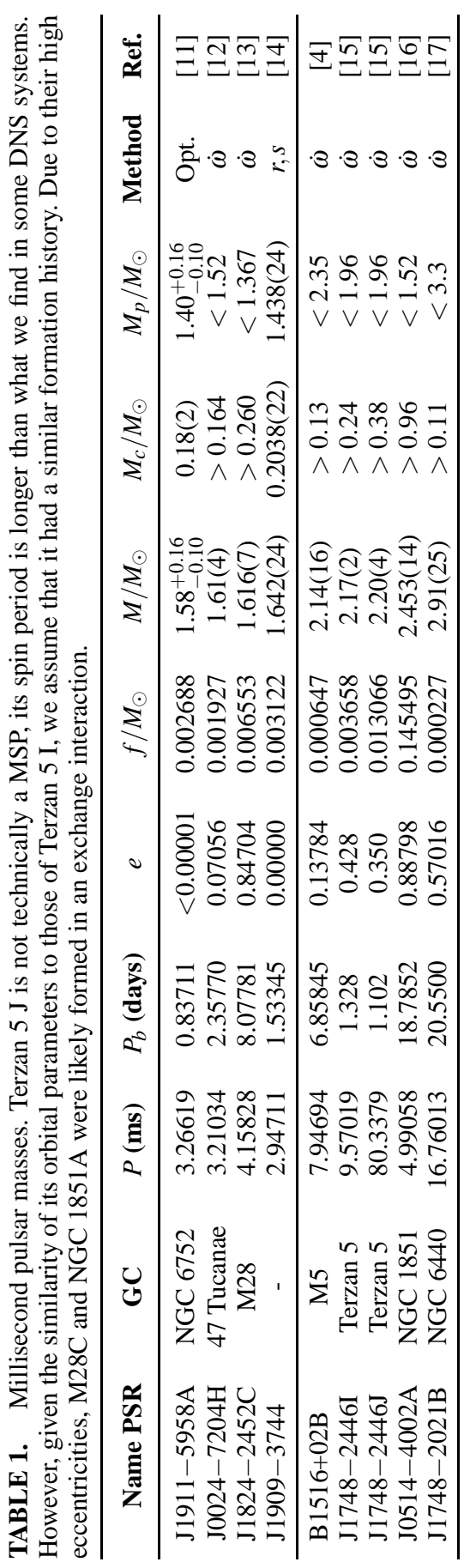

\section{STATISTICAL EVALUATION OF MASS MEASUREMENTS}

A list of mass estimates for millisecond pulsars is presented in Table 1 Most of these were derived for MSPs in GCs. The reason is, of course, than in GCs the MSPWD orbits can be perturbed, resulting in much higher 


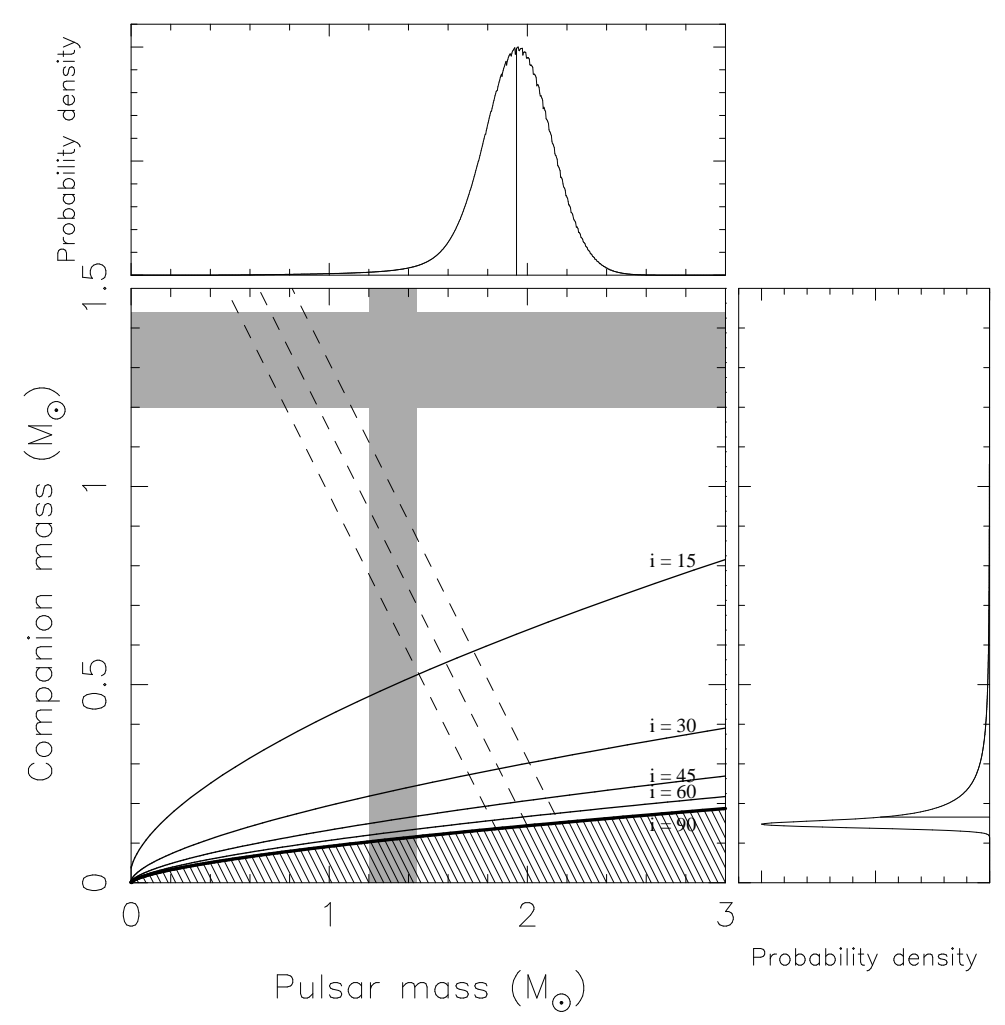

FIGURE 1. Constraints on the masses of M5B and its companion. The hatched region is excluded by knowledge of the mass function and by $\sin i \leq 1$. The diagonal dashed lines correspond to a total system mass that causes a general-relativistic $\dot{\omega}$ equal or within $1-\sigma$ of the measured value. The five solid curves indicate constant inclinations. The gray bars indicate the range of precisely measured neutron star masses (from $\sim 1.20 M_{\odot}$ to $1.44 M_{\odot}$ ). We also display the probability density function for the mass of the pulsar (top) and the mass of the companion (right), and mark the respective medians with vertical (horizontal) lines.

eccentricities than possible in the Galaxy. These eccentricities allow the measurement of post-Keplerian (PK) effects like $\dot{\omega}$. Eventually other PK effects will be measurable, like the Einstein delay $(\gamma)$. However, GC pulsars are generally rather faint, therefore such measurements will require some time.

For that reason, and as in the case of M5B, most of the estimates in Table 1 are based on measurements of $\dot{\omega}$ only. These are "incomplete" measurements, in the sense that one more PK parameter is necessary to have an unambiguous determination of the pulsar mass. Nevertheless, unambiguous upper limits for the mass of the pulsar and lower limits for the mass of the companion can always be obtained in these cases. Furthermore, in systems where the mass function is small (as for M5B) there is a much greater probability of most of the mass of the binary belonging to the pulsar itself (see above).

From Table 1 we can see that the assumption that the $\dot{\omega}$ is relativistic yields for M5B the largest neutron star mass presently known, with the possible exception of PSR J1748-2021B (NGC 6440B, see Paulo Freire's contribution on the new pulsars in NGC 6440 and NGC 6441 in these Proceedings).

\section{Statistical evidence for high neutron star masses}

One of the interesting features of Table 1 is that as the total binary mass increases, the mass function does not increase, with a single exception: PSR J0514-4002A (see Paulo Freire's contribution on NGC 1851A in these Proceedings). This system has a highly eccentric orbit that is thought to have resulted from an exchange interaction [18]. If the increase in total mass for the other systems was due solely to an increase in the mass of the companion, then there should be a more general trend to higher mass functions, i.e., PSR J0514-4002A should be the rule among the massive binaries, not the exception.

It could happen that the separation between the "light" and "massive" binaries (shown by the horizontal line in Table 11) is due merely to a significant classical contribution to $\dot{\omega}$ in the latter group. This could explain all the anomalously high masses produced by the $\dot{\omega}$ estimates. However, it is unlikely that the extra classical contributions have exactly the values required to make the mass estimates nearly identical to each other. Furthermore, a 
possible cause of an extra contribution to $\dot{\omega}$ is tidal effects due to an extended companion. If the presence of extended companions were to explain the large measured values of $\dot{\omega}$, then it should also lead on average to tidal circularization of the orbits of the "massive" binaries, particularly for those with shorter orbital periods (even if that is not guaranteed in individual cases, like M5B, which could have been perturbed recently). In reality, the "massive" binaries are not less eccentric than the "light" binaries.

\section{Statistical combination of mass pdfs}

The mass pdfs for the 7 MSPs in Table 1 with $\dot{\omega}$ measurements were calculated as described above for M5B, and displayed graphically in Fig. 2. The mass medians for Ter 5 I, J and NGC 6440B are 1.87, 1.76 [15] and 2.73 [17] respectively. All these pdfs have long, lowprobability tails towards the low masses, corresponding to improbably low orbital inclinations.

Combining the pdfs for the masses of Ter $5 \mathrm{I}$ and $\mathrm{J}$, [15] reached the conclusion that at least one of these is more massive than 1.48 and $1.68 M_{\odot}$ with $99 \%$ and $95 \%$ confidence levels. Combining those pdfs with the mass pdf for M5B, the probability that all of these pulsars are less massive than 1.72 and $1.79 M_{\odot}$ is only 1 and $5 \%$ respectively. These limits introduce some of the most stringent constraints to date on the bulk behavior of cold, super-dense matter. They exclude many of the "soft" equations of state that have been proposed to model that behavior [19].

From NGC 6440B alone, we can derive a 99\% probability for that pulsar having a mass larger than $2.01 M_{\odot}$, which, if confirmed, would be an even tighter constraint to the EOS. If all $\dot{\omega}$ s are relativisitc, the probability of all these massive binaries having "normal" $\left(1.2<M_{p}<\right.$ $\left.1.44 M_{\odot}\right)$ masses is $9 \times 10^{-9}$.

\section{FORMATION}

The mass pdfs in Fig. 2] suggest that the distribution of MSP masses is bi-modal, with NGC 6440B as a supermassive outlier. PSR J0514-4002A and the MSPs in the "light" $\left(M<2 M_{\odot}\right)$ binaries have masses smaller than $1.5 M_{\odot}$, i.e., they are not significantly more massive than mildly recycled neutron stars, despite having spin frequencies of hundreds of Hz. In particular, the case of M28 C shows that MSPs can be recycled by accreting $<0.15 M_{\odot}$ from their companions. Other MSPs are significantly more massive, it is not clear why they are so.

It could happen that they were born that way. A bimodal (or tri-modal) distribution like that of Fig. 2 is exactly what is predicted by hydrodynamical core collapse simulations [20]: stars below $\sim 18 M_{\odot}$ are expected to form $\sim 1.20-1.35 M_{\odot}$ NSs. Stars with masses between $18-20 M_{\odot}$ form $1.8 M_{\odot}$ NSs. Above $20 M_{\odot}$, stars experience partial fall-back of material that can significantly increase the mass of the remnant, making it either a super-massive NS or, if its mass is above the maximum stable neutron star mass, a black hole.

This possibility raises the question of why such massive NSs have never been found in the 9 known DNS systems (which have a total of $18 \mathrm{NSs}$ ). Given the narrow range of progenitor masses $\left(18-20 M_{\odot}\right)$, it is possible that massive NSs are relatively rare. However, $50 \%$ of the NSs in eccentric binary MSPs in Table 1 are massive.

It is possible that all these NSs started instead with similar masses. In the case of MSPs, the accretion episode is much longer than for the recycled pulsars in DNS systems, with a potentially (but not necessarily) larger mass transfer. This is a natural explanation for why we only see massive NSs as MSPs but not in DNS systems. If this was the case, we should then expect that the more massive MSPs, having accreted more mass and angular momentum, should spin faster than the less massive MSPs. Table 1 shows that the opposite is true: the more massive MSPs spin more slowly $(v<125 \mathrm{~Hz})$ than the less massive MSPs $(v>200 \mathrm{~Hz})$. More statistics are needed to verify the significance of this relation; but if it holds, then there might be two MSP recycling mechanisms, one of them transmitting more angular momentum and the other more mass. If, on the other hand, neutron stars start with different masses, and the more massive NSs have a higher moment of inertia, then transferring the same amount of angular momentum to a massive NS will cause a smaller increase in the spin frequency.

\section{ACKNOWLEDGMENTS}

The Arecibo Observatory, a facility of the National Astronomy and Ionosphere Center, is operated by Cornell University under a cooperative agreement with the National Science Foundation. I thank my collaborators in the GC pulsar searches over the years: many were students, like Steve Bégin, Ryan Lynch, Jeniffer Katz, Lucy Frey and Ben Sulman, others were a bit more experienced, like Andrew G. Lyne, Richard N. Manchester, Vicky Kaspi, Alex Wolszczan and Yashwant Gupta. Special thanks go to Fernando Camilo, Michael Kramer, Duncan Lorimer, Ingrid Stairs, Jason Hessels and, of course, Scott Ransom. 


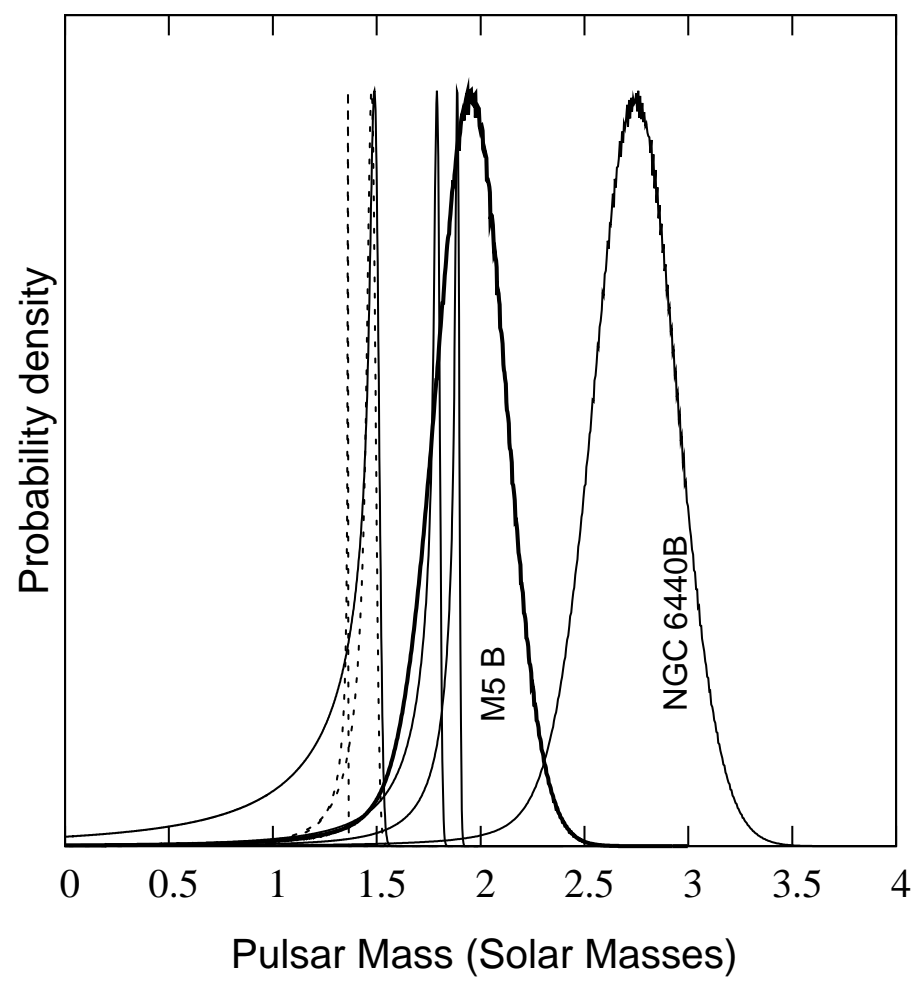

FIGURE 2. Probability distribution functions (pdfs) for seven of the MSPs in Table 1 The mass pdfs of the MSPs in the least massive binaries (those with $M<2 M_{\odot}$ ) are represented by the dashed curves. Despite the limitation of being calculated solely from $\dot{\omega}$, the pdfs capture well the peak in NS mass that is known to occur at $1.2-1.4 M_{\odot}$, suggesting that the peak at $1.8-2.0 M_{\odot}$ is a also a real feature.

\section{REFERENCES}

1. A. Wolszczan, S. Anderson, S. Kulkarni, and T. Prince, Two millisecond pulsars in M5, IAU Circular 4880 (1989).

2. F. R. Rasio, and D. C. Heggie Ap.J., 445, L133-L135 (1995).

3. S. B. Anderson, A. Wolszczan, S. R. Kulkarni, and T. A. Prince Ap.J., 482, 870-873 (1997).

4. P. C. C. Freire, A. Wolszczan, M. van den Berg, and J. W. T. Hessels Ap.J., submitted (2007).

5. J. W. T. Hessels, S. M. Ransom, I. H. Stairs, V. M. Kaspi, and P. C. C. Freire, Ap.J., 670, 363-378 (2007).

6. A. Dowd, W. Sisk, and J. Hagen, "The WAPP - Wideband Arecibo Pulsar Processor," in Pulsar Astronomy - 2000 and Beyond, IAU Colloquium 177, edited by M. Kramer, N. Wex, and R. Wielebinski, Astronomical Society of the Pacific, San Francisco, 2000, pp. 275-276.

7. J. H. Taylor, Philosophical Transactions of the Royal Society of London, A 341, 117-134 (1992).

8. E. M. Standish, JPL Planetary and Lunar Ephemerides, DE405/LE405, Memo IOM 312.F-98-048, JPL, Pasadena, 1998, http://ssd.jpl.nasa.gov/iau-comm4/de405iom/de405iom.pdf

9. A. J. Faulkner, M. Kramer, A. G. Lyne, R. N. Manchester, M. A. McLaughlin, I. H. Stairs, G. Hobbs, A. Possenti, D. R. Lorimer, N. D'Amico, F. Camilo, and M. Burgay, Ap.J. Lett. 618, L119-L122 (2005).
10. J. M. Weisberg, and J. H. Taylor, "The Relativistic Binary Pulsar B1913+16," in Radio Pulsars, edited by M. Bailes, D. J. Nice, and S. Thorsett, Astronomical Society of the Pacific, San Francisco, 2003, pp. 93-98.

11. C. G. Bassa, M. H. van Kerkwijk, D. Koester, and F. Verbunt Ap.J., 456, 295-304 (2006).

12. P. C. Freire, F. Camilo, M. Kramer, D. R. Lorimer, A. G. Lyne, R. N. Manchester, and N. D'Amico M.N.R.A.S., 340, 1359-1374 (2003).

13. S. Bégin, S. M. Ransom, P. C. C. Freire, I. H. Stairs, J. W. T. Hessels, J. Katz, V. Kaspi, and F. Camilo, Ap.J., in preparation (2007).

14. B. A. Jacoby, A. Hotan, M. Bailes, S. Ord, and S. R. Kuklarni, Ap.J. Lett., 629, L113-L116 (2005).

15. S. M. Ransom, J. W. T. Hessels, I. H. Stairs, P. C. C. Freire, F. Camilo, V. M. Kaspi, and D. L. Kaplan, Science 307, 892-896 (2005).

16. P. C. C. Freire, S. M. Ransom, and Y. Gupta, Ap.J., 662, 1177-1182 (2007).

17. P. C. C. Freire, S. M. Ransom, S. Bégin, I. H. Stairs, J. W. T. Hessels, L. H. Frey, and F. Camilo, Ap.J., in press, http://arxiv.org/abs/0711.0925

18. P. C. Freire, Y. Gupta, S. M. Ransom, and C. H. Ishwara-Chandra, Ap.J. Lett., 606, L53-L56 (2004).

19. J. M. Lattimer, and M. Prakash, Phys. Rep. 442, 109-165 (2007).

20. Timmes, F. X., Woosley, S. E., \& Weaver, T. A. 1996, Ap.J., 457, 834-843. 